\title{
Electronic reminders for pathologists promote recognition of patients at risk for Lynch syndrome: cluster-randomised controlled trial
}

\author{
L. I. Overbeek • R. P. Hermens • J. H. van Krieken • \\ E. M. Adang • M. Casparie • F. M. Nagengast • \\ M. J. Ligtenberg $\cdot$ N. Hoogerbrugge • \\ MIPA study group
}

Received: 8 February 2010 /Accepted: 11 March 2010 /Published online: 9 April 2010

(C) The Author(s) 2010. This article is published with open access at Springerlink.com

\begin{abstract}
We investigated success factors for the introduction of a guideline on recognition of Lynch syndrome in patients recently diagnosed with colorectal cancer (CRC) below age 50 or a second CRC below age 70. Pathologists were asked to start microsatellite instability (MSI) testing and report to surgeons with the advice to consider genetic counselling when MSI test or family history was positive. A multicentre cluster-randomised controlled trial (ClinicalTrials.gov, number NCT00141466) was performed in 12 pathology laboratories (clusters), serving 29 community hospitals. All received an introduction to the new guideline. In the intervention group, surgeons received education and
\end{abstract}

The MIPA study group consists of the authors and individuals from the Radboud University Medical Centre (B. Gordijn, R.P.T.M. Grol, K.M. Landsbergen, M.F. Niermeijer, H.G. Brunner and P. van Duijvendijk en R.P. Akkermans) and from the University Medical Centre Groningen (H. Hollema, J.H. Kleibeuker and R.H. Sijmons).

L. I. Overbeek $\cdot$ M. J. Ligtenberg $\cdot$ N. Hoogerbrugge $(\square)$

Department of Human Genetics 849,

Radboud University Nijmegen Medical Centre,

P.O. Box 9101, 6500 HB Nijmegen, The Netherlands

e-mail: n.hoogerbrugge@antrg.umcn.nl

L. I. Overbeek $\cdot$ R. P. Hermens

Centre for Quality of Care Research,

Radboud University Nijmegen Medical Centre,

Nijmegen, The Netherlands

J. H. van Krieken • M. J. Ligtenberg

Department of Pathology,

Radboud University Nijmegen Medical Centre,

Nijmegen, The Netherlands

\section{E. M. Adang}

Department of Epidemiology,

Biostatistics and Health Technology Assessment,

Radboud University Nijmegen Medical Centre,

Nijmegen, The Netherlands tumour test result reminders; pathologists were provided with inclusion criteria cards, an electronic patient inclusion reminder system and feedback on inclusion. Two hundred sixty-six CRC patients were eligible for recognition as at risk for Lynch syndrome. The actual recognition was $18 \%$ more successful in the intervention as compared to the control arm (77\% (120 of 156) compared to 59\% (65 of $110)$ ), with an adjusted odds ratio $(\mathrm{OR})=2.8(95 \%$ confidence interval (CI) 1.1-7.0). The electronic reminder system for pathologists was most strongly associated with recognition of high-risk patients, $\mathrm{OR}=4.2$ (95\% CI 1.7 10.1). An electronic reminder system for pathologists

M. Casparie

PALGA Registry for Pathology,

Utrecht, The Netherlands

\section{F. M. Nagengast}

Department of Gastroenterology,

Radboud University Nijmegen Medical Centre,

Nijmegen, The Netherlands

N. Hoogerbrugge

Department of Medical Oncology,

Radboud University Nijmegen Medical Centre,

Nijmegen, The Netherlands 
appeared effective for adherence to a new complex guideline and will enhance the recognition of Lynch syndrome.

Keywords Colorectal cancer $\cdot$ HNPCC $\cdot$ Lynch syndrome MSI testing $\cdot$ Bethesda criteria

\section{Introduction}

In this era of rapidly increasing knowledge on hereditary cancer, much new evidence needs to be introduced in clinical practice, often in multidisciplinary teams including surgeons, pathologists and clinical geneticists. A commonly used method to achieve awareness is the development of guidelines for clinical practice. In recent years, many such guidelines have been published. However, approximately $30-40 \%$ of patients do not receive care according to present scientific evidence, and about $20-25 \%$ of care provided is not needed or is potentially harmful [1]. At present, little is known about effective strategies for the implementation of new guidelines in multidisciplinary teams of second-line medical care $[2,3]$.

Lynch syndrome, previously referred to as hereditary non-polyposis colorectal cancer [4], is the most common type of hereditary CRC and is caused by germ line inactivation of one of four DNA mismatch repair genes [5]. Although Lynch syndrome only accounts for up to $5 \%$ of CRCs [6-9], identifying Lynch syndrome is highly relevant because surveillance substantially reduces morbidity and mortality in family members carrying a mismatch repair gene mutation [10]. The identification of Lynch syndrome starts with the recognition of patients at risk for Lynch syndrome. Traditionally, this is done by the occurrence of CRC in the family. However, by using family history, only a small proportion of the expected number of patients at risk for Lynch syndrome is identified [11-14]. This is due to small families, unawareness by the patients of their own family history and suboptimal registration of family history of cancer by surgeons and gastroenterologists [11-14]. Recognising patients at risk for Lynch syndrome can effectively be improved by an additional procedure using a molecular genetic test on tumour DNA of a CRC, the so-called microsatellite instability (MSI) testing [7, 9, 15, 16]. MSI is a hallmark of a defective mismatch repair system, which results in differences in length of stretches of simple repeat sequences, called microsatellites, between normal and tumour DNA. In Lynch syndrome, virtually all CRCs show MSI. Although MSI may also occur in sporadic CRC diagnosed at relatively high age, in the group of patients suspected for Lynch syndrome, the MSI is predominantly due to genetic susceptibility [17]. Once patients at risk are identified, this will lead to identification of more patients with Lynch syndrome in a highly cost-effective way $[15,18]$.

A new guideline was developed using MSI testing to improve recognition of patients at risk for Lynch syndrome by a multidisciplinary team of surgeons, pathologists and clinical geneticists. In this MSI-testing-indicated-by-a-pathologist (MIPA) procedure [15], pathologists initiate MSI testing in a selection of recently diagnosed patients with one of the following criteria: (1) CRC diagnosed before age 50, (2) second CRC before age 70, (3) CRC and a Lynch syndrome associated cancer before age 70 or (4) a colorectal adenoma with high-grade dysplasia before the age of 40 years $[15,19$, 20]. Pathologists report the MSI test result to the surgeon with the advice to consider referral for genetic counselling in an outpatient clinic for hereditary cancer when the MSI test was positive or the family history remained suspicious for hereditary CRC.

This guideline was proven to be feasible and costeffective, includes modern molecular techniques for pathologists and requires more communication between surgeons, pathologists and clinical geneticists $[15,21]$. To introduce the MIPA procedure in community hospitals, an implementation strategy was developed, tailored to barriers found in a feasibility study [21]. The aim of the present study was to determine the effects and costs of this implementation. The secondary aim was to determine which elements of the implementation were particularly successful.

\section{Patients and methods}

\section{Study design}

The MIPA study was a cluster-randomised controlled trial in 12 pathology laboratories and the 29 community hospitals in their catchment area, examining the effectiveness of a tailored implementation strategy for the MIPA procedure compared to standard implementation. The Committee on Research Involving Human Subjects Region ArnhemNijmegen approved the study and waived the need for written consent from patients (project approval 2004/156).

\section{Patients}

Patients recently diagnosed with $\mathrm{CRC}$ were eligible for inclusion. Selection criteria for MSI testing, the MIPA criteria, were derived from the internationally used Bethesda guidelines as described [15]. The following tumours were included as associated with Lynch syndrome: carcinomas of endometrium, ovary, small bowel, stomach, biliary tract, upper urinary tract and sebaceous gland [5]. The nationwide electronic registry for pathology in the Netherlands, PALGA [22], was used for the identification 
of tumours that fulfilled the selection criteria. After closing the study, 279 patients turned out to have been eligible for inclusion between September 2005 and December 2006.

\section{Randomisation}

Randomisation was done at the level of the pathology laboratories (clusters).

\section{Intervention}

All surgeons and pathologists received written information on the new procedure for recognition of Lynch syndrome, as published articles and a guideline. In the intervention group, pathologists were also provided with electronic patient inclusion reminders (monthly), feedback on inclusion (every 3 month) and supporting materials such as small cards with the selection criteria, a list to register MSIpositive tumours and a folder to save information of MSI applications. Surgeons received a $1-\mathrm{h}$ interactive smallscale education session with a lecture and discussion concerning how and when to tell a patient about a positive MSI test result. Surgeons also received reminders in the patient record, consisting of a sticker with a checklist of three items: (a) MSI result (positive or negative), (b) test result discussed with patient (yes or no) and (c) patient referred to cancer genetics clinic (yes or no).

\section{Outcome measures}

The primary outcome measure was the percentage of patients recognised to be at risk for Lynch syndrome. Recognition was defined as (1) patients with a positive MSI result who were referred to genetic counselling, or (2) patients with a negative MSI result, or (3) patients who were referred to genetic counselling without prior MSI application by a pathologist. The secondary outcome measures were the effectiveness of the elements directed to pathologists and surgeons [2].

\section{Costs of implementation strategy}

To gather data about the costs of the elements of the implementation strategy, surgeons, pathologists and the research team provided time estimates. These volumes were multiplied by standardised unit costs [23]. Because these unit costs were established in 2003, they were adjusted to 2007 by using the Consumer Price Index as published by the Central Bureau of Statistics in the Netherlands (StatLine, www.statline.cbs.nl). Overhead costs were calculated as $32 \%$ of the direct costs to approximate overhead in health care. All costs were measured on a per patient basis.
Statistical analysis

Results are reported according to guidelines for reporting cluster-randomised trials [24]. To guarantee that the laboratories and their hospitals in the intervention group did not differ in pre-randomisation performance from the laboratories and hospitals in the control group regarding the outcome measure, we retrospectively analysed in both groups 'the percentage of patients who were referred to genetic counselling without prior MSI application by a pathologist'. This concerned patients who were recently diagnosed with $\mathrm{CRC}$ in the participating pathology laboratories between April and December 2004. We measured only this part of the primary outcome measure because MSI testing was not initiated by the pathologists before the start of the intervention. To examine the rate of patients referred to genetic counselling, all Dutch cancer genetics clinics were asked to check whether the patients selected by PALGA had visited their hospital. The percentage of patients who received genetic counselling was $30.2 \%$ (95\% confidence interval (CI) $18.5-41.8 \%$ ) in the intervention and $30.4 \%$ (95\% CI $17.9-42.8 \%)$ in the control group, so the pre-randomisation performance of both groups was very comparable. To ascertain successful elements of the implementation strategy, the experiences of pathologists and surgeons with the elements scored on a six-point Likert scale were dichotomised into positive or negative experience. Odds ratios were calculated to describe univariate associations between each element of the implementation strategy and the secondary outcome measure. Statistical significance was set at 5\%. Analyses were performed with the SAS system for Windows V8.2.

\section{Results}

Characteristics of clusters and participants

Two hundred seventy-nine patients were eligible for recognition at risk for Lynch syndrome. In total, 13 patients were excluded from analysis because insufficient tumour material was available $(n=4)$; patients had been diagnosed before with either Lynch syndrome $(n=5)$ or with familial adenomatous polyposis $(n=4)$. Therefore, the study population consisted of 266 patients: 156 patients in the intervention arm and 110 in the control arm, treated in 17 and 12 community hospitals, respectively. The characteristics of clusters and participants in the intervention and the control arm are summarised in Table 1.

Effects of implementation strategy

In Fig. 1, a flow diagram of the recognition of patients at risk for Lynch syndrome is given. Patients were recognised 
Table 1 Characteristics of study population

Intervention

$\begin{array}{ll}6 & 6 \\ 17 & 12 \\ 156 & 110 \\ 118(76 \%) & 78(71 \%) \\ 26(17 \%) & 18(16 \%) \\ 7(4 \%) & 2(2 \%) \\ 5(3 \%) & 12(11 \%) \\ 48.2(\mathrm{SD}=9.8) & 46.2(\mathrm{SD}=9.7)\end{array}$

Control

\section{Clusters:}

Pathology laboratories

Hospitals

\section{Patients:}

CRC below the age of 50 years

Second CRC below the age of 70 years

$\mathrm{CRC}$ and extracolonic cancer below the age of 70 years $^{\mathrm{a}}$

Colorectal adenoma with high-grade dysplasia below the age of 40 years Age at diagnosis

${ }^{a}$ Extracolonic cancers: malignancies associated with Lynch syndrome (endometrial, ovaries, small bowel, stomach, sebaceous gland, biliary tract and upper urinary tract)

as at risk for Lynch syndrome significantly more often in the intervention arm compared to the control arm, 120 out of $156(77 \%)$ patients and 65 out of $110(59 \%)$ patients, respectively, with a for clustering adjusted $\mathrm{OR}=2.8(95 \%$ CI 1.1-7.0). Pathologists in the intervention arm applied MSI testing to 116 out of 156 patients (74\%) and pathologists in the control arm to 56 out of 110 patients (51\%). This $23 \%$ difference in applying MSI testing between the intervention and control arm was significant as well, adjusted $\mathrm{OR}=4.1$ (95\% CI 1.3-13.2). Surgeons from the intervention arm referred as many patients with an MSI-positive tumour to genetic counselling as those in the control arm: 14 out of 19 (74\%) patients and 7 out of 11 (64\%) patients, respectively, OR=1.6 (95\% CI 0.09-7.9).

Successful elements of implementation strategy

Five out of six pathologists experienced the electronic patient inclusion reminder system useful, but none of the pathologists thought that the feedback on inclusion every 3 months improved application of MSI testing. The pathologist who scored our electronic reminders as not effective used a self-developed electronic patient inclusion reminder system and actually meant that receiving our

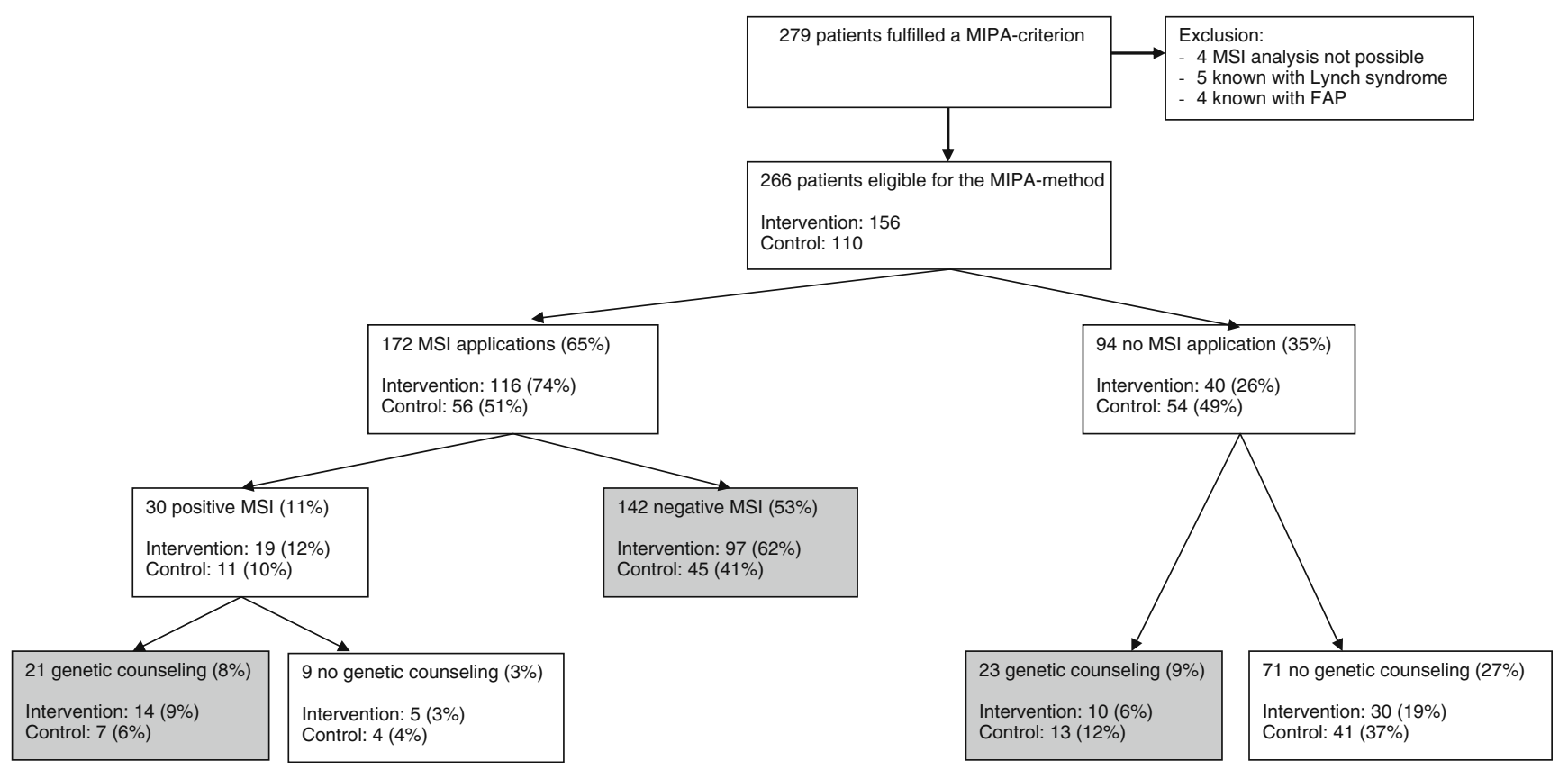

Fig. 1 Flow diagram of the recognition of patients at risk for Lynch syndrome 
electronic reminders was not of additional value. Furthermore, it appeared that two pathology laboratories of the control arm had developed a reminder system very similar to the one provided in the intervention arm by themselves. The availability of an electronic patient inclusion reminder system was highly associated with the detection of patients at risk for Lynch syndrome: 156 out of 204 (76\%) and 29 out of $62(47 \%)$ with and without an electronic patient inclusion reminder system, respectively (clustering adjusted $\mathrm{OR}=4.2$ (95\% CI 1.7-10.1)). All pathologists agreed with the need for a reminder system because only a small subset (about 13\%) of patients with CRC fulfils the criteria for MSI testing [15] and therefore referring a case for MSI testing can easily be forgotten. Nevertheless, MSI testing was applied in only $75 \%$ of eligible tumours from pathology laboratories with an electronic reminder system. According to interviews with the pathologists, barriers to application of MSI testing were time constraints and absence of the pathologist who was primarily responsible for application of MSI testing. Because the intervention directed to surgeons was not significantly effective, it was not possible to determine successful elements at this level.

Costs of implementation strategy

The costs of the elements of the intervention are presented in Table 2. The total costs per patient at risk for Lynch syndrome were $€ 78$ for the intervention and $€ 11$ for the control arm. The implementation and or running costs of the electronic patient inclusion reminder system for pathologists was $€ 27$ per patient at risk.

\section{Discussion}

This study shows that a simple electronic reminder system for pathologists markedly increases adherence to the MIPA procedure, a multidisciplinary guideline for recognition of CRC patients at high risk for Lynch syndrome. With surprisingly little effort and at low costs, multidisciplinary implementation of an entirely novel practice role for pathologists was improved. The efficiency of counselling by a clinical geneticist is greatly enhanced when patients with an MSI-high CRC are being referred for Lynch syndrome. This is due to the fact that especially patients with a positive MSI test are at very high risk to be mutation carriers, whereas a clinical geneticist has little to offer to a young patient with an MSI-negative CRC, unless the presence of polyposis or a positive family history clearly points at a hereditary defect. Our results may have a larger impact on health care because use of an electronic reminder system can increase adherence to guidelines of other hereditary diseases too.

To our knowledge, this is the first study of an implementation strategy designed to improve the recognition of patients at risk for Lynch syndrome by tumour

Table 2 Costs of development and execution of elements in euros

\begin{tabular}{|c|c|c|c|c|}
\hline \multirow[t]{2}{*}{ Elements } & \multicolumn{2}{|l|}{ Total cost per element } & \multirow{2}{*}{$\begin{array}{l}\text { Intervention arm } \\
\text { Costs of execution per } \\
\text { patient }\end{array}$} & \multirow{2}{*}{$\begin{array}{l}\text { Control arm } \\
\text { Costs of execution } \\
\text { per patient }\end{array}$} \\
\hline & Total cost of development & Total cost of execution & & \\
\hline Dissemination of MIPA method & 1,586 & 792 & $1^{\mathrm{b}}$ & $1^{\mathrm{b}}$ \\
\hline Introduction of MIPA method & - & 5,996 & $10^{\mathrm{b}}$ & $10^{\mathrm{b}}$ \\
\hline \multicolumn{5}{|l|}{ Directed to pathologists: } \\
\hline Supporting materials ${ }^{\mathrm{a}}$ & 39 & 164 & $1^{\mathrm{c}}$ & - \\
\hline Electronic reminders & - & 4,260 & $27^{\mathrm{d}}$ & - \\
\hline Feedback & - & 568 & $4^{\mathrm{d}}$ & - \\
\hline \multicolumn{5}{|l|}{ Directed to surgeons: } \\
\hline Education & 2,238 & 6,128 & $20^{\mathrm{c}}$ & - \\
\hline Reminders in surgical record & 373 & 1,520 & $16^{\mathrm{e}}$ & - \\
\hline Total & 4,235 & 19,428 & $78^{\mathrm{b}}$ & $11^{\mathrm{b}}$ \\
\hline
\end{tabular}

See "Costs of implementation strategy" in the "Patients and method" section

${ }^{a}$ Supporting materials include: folder, card with selection criteria, list for calling clinicians

${ }^{\mathrm{b}}$ Total cost of execution of element divided by 600 patients

${ }^{\mathrm{c}}$ Total cost of execution of element divided by 300 patients

${ }^{\mathrm{d}}$ Total cost of execution of element divided by 156 patients

${ }^{\mathrm{e}}$ Total cost of execution of element divided by 39 
testing. One year before the introduction of the MIPA procedure, only $30 \%$ of the patients at risk for Lynch syndrome was recognised as such by the traditional procedure based on signalling familial occurrence of colorectal cancer [25]. This low number is in line with other studies showing that family history is insufficient for recognising patients at risk for Lynch syndrome [11-14]. After introduction of the MIPA procedure in multidisciplinary teams including surgeons and pathologists by written materials, the recognition of patients at risk for Lynch syndrome was substantially higher, namely $59 \%$. In the tailored implementation group, this recognition was even $77 \%$. This difference between control and intervention group is relatively large. In a review including 235 controlled studies, Grimshaw [2] studied the effectiveness of strategies to implement clinical guidelines. Overall, the majority of studies observed modest improvements in care of only $5-10 \%$.

In the present study, the pathologists in the intervention arm obtained an overview of eligible patients only once a month. It is expected that the MSI analysis would be applied even more often, when the pathologist would get a pop-up with the possibility to initiate MSI testing at the moment of authorising the pathology report.

The surgeon-directed intervention led to $74 \%$ referrals of patients with an MSI-positive tumour. This was not significantly larger than in the control group (64\%) probably because of small numbers. It is plausible that a referral rate of $74 \%$ is fairly optimal because not all patients with an MSI-positive tumour are capable or willing to receive genetic counselling at the time colorectal cancer is diagnosed and treated. Our results are in line with previous data showing that $78 \%$ of patients with CRC would accept genetic counselling shortly after diagnosis [26].

Based on the Bethesda criteria, originally four MIPA criteria have been defined. However, only two of these criteria appeared to be fulfilled within our study. Therefore, we suggest restricting the MIPA criteria to the following two criteria: (1) CRC diagnosed before age 50 and (2) second CRC before age 70 .

A limitation of the study is that the patient's visit to a cancer genetics clinic for genetic counselling was measured until 6 months after the CRC diagnosis of the last patient. It is possible that more patients will receive genetic counselling in the near future. However, as it is unlikely that this effect differs between the two groups, this will not affect the conclusion that the intervention leads to a higher rate of adherence to the guideline.

Our findings indicate that a surprisingly simple and cheap electronic patient inclusion reminder system for medical specialists is effective for adherence to a complex multidisciplinary procedure for recognition of Lynch syndrome. The implications for health care can be substantially and are of course not restricted to patients at risk for Lynch syndrome. Our approach can serve as a model for implementation of guidelines in other hereditary diseases.

Acknowledgement The following pathology laboratories and Dutch hospitals are kindly thanked for their cooperation: Meander Medical Centre Amersfoort, Alysis zorggroep Arnhem, HagaZiekenhuis The Hague, Albert Schweitzer Hospital Dordrecht, Catharina Hospital Eindhoven, Medisch Spectrum Twente Enschede, Elkerliek Hospital Helmond, Medical Centre Leeuwarden, Canisius Wilhelmina Hospital Nijmegen, Medical Centre Rijnmond Zuid Rotterdam, Jeroen Bosch Hospital 's-Hertogenbosch and St Elisabeth Hospital Tilburg. The Department of Human Genetics of University Hospital Maastricht, Erasmus MC Rotterdam, University Medical Centre Groningen, University Medical Centre Utrecht and Leiden University Medical Centre are thanked for checking referrals to genetic counselling.

Conflict of interest statement We declare that we have no conflict of interest.

Funding This work was supported by a financial grant from the Netherlands Organisation for Health Research and Development (ZonMW).

Open Access This article is distributed under the terms of the Creative Commons Attribution Noncommercial License which permits any noncommercial use, distribution, and reproduction in any medium, provided the original author(s) and source are credited.

\section{References}

1. Grol R, Grimshaw J (2003) From best evidence to best practice: effective implementation of change in patients' care. Lancet 362:1225-1230

2. Grimshaw JM, Thomas RE, Maclennan G et al (2004) Effectiveness and efficiency of guideline dissemination and implementation strategies. Health Technol Assess 8:1-72

3. Shaw B, Cheater F, Baker R et al (2005) Tailored interventions to overcome identified barriers to change: effects on professional practice and health care outcomes. Cochrane Database Syst Rev 2005:CD005470

4. Jass JR (2006) Hereditary non-polyposis colorectal cancer: the rise and fall of a confusing term. World J Gastroenterol 12:4943-4950

5. Lynch HT, de la Chapelle A (2003) Hereditary colorectal cancer. N Engl J Med 348:919-932

6. Aaltonen LA, Salovaara R, Kristo P et al (1998) Incidence of hereditary nonpolyposis colorectal cancer and the feasibility of molecular screening for the disease. N Engl J Med 338:14811487

7. Barnetson RA, Tenesa A, Farrington SM et al (2006) Identification and survival of carriers of mutations in DNA mismatch-repair genes in colon cancer. N Engl J Med 354:2751-2763

8. Cunningham JM, Kim CY, Christensen ER et al (2001) The frequency of hereditary defective mismatch repair in a prospective series of unselected colorectal carcinomas. Am J Hum Genet 69:780-790

9. Hampel H, Frankel WL, Martin E et al (2005) Screening for the Lynch syndrome [hereditary nonpolyposis colorectal cancer]. N Engl J Med 352:1851-1860 
10. Jarvinen HJ, Aarnio M, Mustonen H et al (2000) Controlled 15year trial on screening for colorectal cancer in families with hereditary nonpolyposis colorectal cancer. Gastroenterology 118:829-834

11. Church J, McGannon E (2000) Family history of colorectal cancer: how often and how accurately is it recorded? Dis Colon Rectum 43:1540-1544

12. Grover S, Stoffel EM, Bussone L et al (2004) Physician assessment of family cancer history and referral for genetic evaluation in colorectal cancer patients. Clin Gastroenterol Hepatol 22:813-819

13. Katballe N, Juul S, Christensen M et al (2001) Patient accuracy of reporting on hereditary non-polyposis colorectal cancer-related malignancy in family members. Br J Surg 88:1228-1233

14. Sijmons RH, Boonstra AE, Reefhuis J et al (2000) Accuracy of family history of cancer: clinical genetic implications. Eur J Hum Genet 8:181-186

15. Kievit W, de Bruin JH, Adang EM et al (2005) Cost effectiveness of a new strategy to identify HNPCC patients. Gut 54:97-102

16. Niessen RC, Berends MJ, Wu Y et al (2006) Identification of mismatch repair gene mutations in young colorectal cancer patients and patients with multiple HNPCC-associated tumours. Gut 55:1781-1788

17. Overbeek LI, Kets CM, Hebeda KM et al (2007) Patients with an unexplained microsatellite instable tumour have a low risk of familial cancer. Br J Cancer 96:1605-1612

18. Vasen HF, van Ballegooijen M, Buskens E et al (1998) A costeffectiveness analysis of colorectal screening of hereditary nonpolyposis colorectal carcinoma gene carriers. Cancer 82:1632-1637
19. Rodriguez-Bigas MA, Boland CR, Hamilton SR et al (1997) A National Cancer Institute Workshop on Hereditary Nonpolyposis Colorectal Cancer Syndrome: meeting highlights and Bethesda guidelines. J Natl Cancer Inst 89:1758-1762

20. Umar A, Boland CR, Terdiman JP et al (2004) Revised Bethesda guidelines for hereditary nonpolyposis colorectal cancer [Lynch syndrome] and microsatellite instability. J Natl Cancer Inst 96:261-268

21. de Bruin JH, Kievit W, Ligtenberg MJ et al (2005) More hereditary intestinal cancer can be detected if patients with colorectal carcinoma that are selected by the pathologist are examined for microsatellite instability. Ned Tijdschr Geneeskd 149:1792-1798

22. Casparie M, Tiebosch AT, Burger G et al (2007) Pathology databanking and biobanking in The Netherlands, a central role for PALGA, the nationwide histopathology and cytopathology data network and archive. Cell Oncol 29:19-24

23. Oostenbrink JB, Bouwmans CAM, Koopmanschap MA, Rutten FFH. (2004) Handleiding voor kostenonderzoek, methoden en standaard kostprijzen voor economische evaluaties in de gezondheidszorg. 2008, College voor zorgverzekeringen

24. Campbell MK, Elbourne DR, Altman DG (2004) CONSORT statement: extension to cluster randomised trials. BMJ 328:702-708

25. Overbeek LI, Hoogerbrugge N, van Krieken JH et al (2008) Most patients with colorectal tumors at young age do not visit a cancer genetics clinic. Dis Colon Rectum 51:1249-1254

26. Porteous M, Dunckley M, Appleton S et al (2003) Is it acceptable to approach colorectal cancer patients at diagnosis to discuss genetic testing? A pilot study. Br J Cancer 89:1400-1402 